\title{
Hypofractionated Palliative Radiotherapy with Concurrent Radiosensitizing Chemotherapy for Advanced Head and Neck Cancer Using the "QUAD-SHOT Regimen"
}

\author{
MAURICIO E. GAMEZ ${ }^{1}$, MANUJ AGARWAL ${ }^{2}$, KENNETH S. HU ${ }^{3}$, JOHN N. LUKENS ${ }^{4}$ and LOUIS B. HARRISON ${ }^{5}$ \\ ${ }^{1}$ Department of Radiation Oncology, Mayo Clinic, Phoenix, AZ, U.S.A.; \\ ${ }^{2}$ Department of Radiation Oncology, University of Maryland School of Medicine, Baltimore, MD, U.S.A.; \\ ${ }^{3}$ Department of Radiation Oncology, New York University, New York, NY, U.S.A.; \\ ${ }^{4}$ Department of Radiation Oncology, University of Pennsylvania, Philadelphia, PA, U.S.A.; \\ ${ }^{5}$ Department of Radiation Oncology, Moffitt Cancer Center, Tampa, FL, U.S.A.
}

\begin{abstract}
Aim: To analyze the outcomes using the hypofractionated palliative radiotherapy regimen " $Q U A D$ Shot" with concurrent radiosensitizing chemotherapy for advanced head and neck cancer. Materials and Methods: We analyzed twenty-one patients with newly-diagnosed or recurrent head and neck cancer treated with palliative hypofractionated concurrent chemoradiation using the QUAD-Shot regimen. Results: All patients received at least one cycle of $R T$, with sixteen patients (76\%) completing all three cycles. $85.7 \%$ of patients had objective response to therapy with five patients (23.8\%) demonstrating complete response $(C R)$ and thirteen patients $(61.9 \%)$ demonstrating partial response (PR). Palliation of symptoms was achieved in all (100\%) of the sixteen patients that completed the three cycles. Median overall survival and median progression-free survival were 7 and 4 months, respectively. Conclusion: QUAD-Shot palliative radiation therapy coupled with radiosensitizing chemotherapy is efficacious and well-tolerated in patients with newly-diagnosed or recurrent head and neck cancer not amenable to curative therapy.
\end{abstract}

While considerable progress has been made in the realm of curative-intent treatment of locally advanced head and neck cancer, there is considerable debate regarding optimal palliative regimens for this disease (1). Such cases represent a minority of patients who are not amenable to radical

Correspondence to: Mauricio E. Gamez, MD, Mayo Clinic Arizona (Proton Cancer Center), 5881 E. Mayo Boulevard, Phoenix, AZ 85054, U.S.A. Tel: 480-342-1262, Fax: 480-342-3972, email: Gamez.mauricio@mayo.edu

Key Words: Quad-Shot, palliative, radiosensitizing chemotherapy, head and neck. therapy due to comorbidities, bulkiness of the tumor and/or stage of the disease. With an increase in cancer burden in an aging population, such cases are more frequent and optimization of therapy is of considerable importance. If left unchecked, patients may have progressive dysphagia, upper airway obstruction, voice quality impairment, local and referred pain, aspiration, bleeding and malnutrition. Treatment in this context presents the challenge of maintaining balance between tumor regression and avoidance of inducing toxicity without reducing quality of life in their limited longevity.

In the last decade a handful of institutions have published their experience with palliative-intent radiotherapy with a variety of fractionation schemes. Treatment schedules have ranged from stereotactic radiation to accelerated hypofractionation. Response rates (partial and complete) have ranged from $47-86 \%$, with complete responses in 0 $45 \%$ of patients and low rates of grade 3 toxicity and improved quality of life in $44-85 \%$ (2-7).

A popular approach in North America is the "QUADShot" regimen, in which $3.7 \mathrm{~Gy}$ are delivered twice a day for two consecutive days and repeated every three or four weeks for a total dose of 4,440 cGy in three cycles. This short cycling schedule was first introduced by RTOG 85-02 as a palliative prescription for numerous body sites and allows flexibility and convenience in a population of patients whose condition may deteriorate rapidly (8-9). When compared to other fractionation schedules, it has been associated with better treatment compliance and less treatment-induced toxicity (1). It is thought to be well applied to the head and neck setting, as it delivers a biologically equivalent dose just below the threshold for producing mucositis and the interval of separation is sufficient for depleted mucosal stem cells to repopulate before the next cycle (4). Efficacy of therapy is easily assessed on physical examination and is repeated in 
responders. In a sentinel phase II study of 30 patients, $54 \%$ of patients had an objective response and $44 \%$ of patients noted an improvement in quality of life (4).

While results with hypofractionated radiotherapy in this population of patients have been documented, the experience utilizing radiosensitizing systemic therapy has rarely been reported. Response rates from palliative chemotherapy regimens have ranged from 15-40\% (10-15). Different chemotherapeutic agents have been demonstrated to act as potent radiosensitizers, particularly platinum agents by causing a $\mathrm{G}_{2} / \mathrm{M}$ cell cycle block (16-22). Its addition to palliative radiotherapy is the next logical step to improve outcomes for these patients. However, there is concern regarding the ability of such patients to tolerate an added modality. A single institution phase II study including 19 patients ( 7 with head and neck malignancies) demonstrated that the addition of paclitaxel to palliative radiation with "QUAD-Shot" fractionation was well-tolerated with improved tumor response rates and symptom palliation (9).

The present study reports our experience with hypofractionated palliative local radiotherapy utilizing the "QUAD-Shot" regimen with concurrent radiosensitizing systemic therapy at a comprehensive cancer center with physicians specialized in the care of patients with head and neck malignancies. We evaluated tumor response, palliation of symptoms, tolerability of treatment and toxicity.

\section{Materials and Methods}

Design. This study is a retrospective analysis of patients with symptomatic, newly diagnosed or recurrent locally advanced head and neck cancer not amenable to curative intent local therapy. Study approval was obtained from our institution's Institutional Review Board and Ethics Committee.

Eligible patients had histologically or cytologically proven malignancy from a locally advanced primary of the head and neck region or large nodal metastasis from an unknown primary suspected to be of head and neck origin. They were ineligible for curative intent therapy due to extent/bulkiness of disease, extensive comorbidity, or distant metastatic disease with an estimated life expectancy of at least three months. Patient's tumor stage, performance status and weight were recorded at first consultation with Radiation Oncology. Patients were required to have an Eastern Cooperative Oncology Group (ECOG) performance status of 0-3 at enrollment and be mentally competent to sign an informed consent. Patients with an active infection or either radiation or chemotherapy within 30 days were excluded.

Pre-treatment evaluation included a medical history, physical examination, complete blood cell count (CBC), complete metabolic profile (CMP), and appropriate radiographic studies to assess disease extent. Patients that met eligibility criteria between the period of January 2005 to June of 2013 were included in the analysis.

Radiotherapy. All treatments were delivered by specialized head and neck radiation oncologists. Patients were simulated in supine position with head and neck cast immobilization prior to each
Table I. Treatment scheme

\begin{tabular}{lccc}
\hline QUAD SHOT & $\begin{array}{c}\text { Cycle 1 } \\
\text { (week 1) } \\
\text { RT + CT }\end{array}$ & $\begin{array}{c}\text { Cycle 2 } \\
\text { (week 4) } \\
\text { RT + CT }\end{array}$ & $\begin{array}{c}\text { Cycle 3 } \\
\text { (week 7) } \\
\text { RT + CT }\end{array}$ \\
\hline Day 1 Fraction 1 & $3.7 \mathrm{~Gy}$ & $3.7 \mathrm{~Gy}$ & $3.7 \mathrm{~Gy}$ \\
Day 1 Fraction 2 & $3.7 \mathrm{~Gy}$ & $3.7 \mathrm{~Gy}$ & $3.7 \mathrm{~Gy}$ \\
Day 2 Fraction 1 & $3.7 \mathrm{~Gy}$ & $3.7 \mathrm{~Gy}$ & $3.7 \mathrm{~Gy}$ \\
Day 2 Fraction 2 & $3.7 \mathrm{~Gy}$ & $3.7 \mathrm{~Gy}$ & $3.7 \mathrm{~Gy}$ \\
Total Dose & $14.8 \mathrm{~Gy}$ & $29.6 \mathrm{~Gy}$ & $44.4 \mathrm{~Gy}$ \\
\hline
\end{tabular}

RT: Radiation Therapy; CT: Chemotherapy.

radiation cycle. Gross primary and nodal tumoral volume (GTV) were delineated by the treating radiation oncologist using diagnostic imaging and clinical examination findings. The clinical target volume (CTV) was equivalent to the GTV, except in areas of uncertainty where additional expansions were applied. For all patients a $0.5-1.0 \mathrm{~cm}$ margin for the planning target volume (PTV) was added depending on setup uncertainty and available image guidance during treatment. Surface bolus was used for nodal disease with skin invasion or fungation. No elective nodal coverage was included in the treatment volumes.

Radiation therapy was delivered using the "QUAD-Shot" regimen, as previously described by RTOG 85-02. Two daily fractions of 3.7Gy were delivered each day separated by an interval of at least $6 \mathrm{~h}$ for two consecutive days, for a dose of 14.8Gy over four fractions. This was repeated every three weeks for a total of three cycles and a total dose of $44.4 \mathrm{~Gy}$ (Table I), as tolerated, provided there was no tumor progression or significant acute toxicity. The biologically equivalent dose (BED) for each quad shot cycle was $20.2 \mathrm{~Gy}_{10}$ and $33.0 \mathrm{~Gy}_{3}$ for tumor and late reacting tissues (EQD2 $16.8 \mathrm{~Gy}_{10}$ and $19.8 \mathrm{~Gy}_{3}$ ), respectively. The BED for two cycles $40.5 \mathrm{~Gy}_{10}$ and $66.1 \mathrm{~Gy}_{3}$ and for three cycles was $60.8 \mathrm{~Gy}_{10}$ and 99.1 $\mathrm{Gy}_{3}$, assuming no repopulation between two fractions.

All treatments were delivered using a megavoltage linear accelerator with $6 \mathrm{MV}$ photons. 3D conformal technique was initially used for treatment delivery until the adoption of Intensity Modulated Radiation Therapy (IMRT) and later with image guidance (IGRT). All patients were examined before and after each treatment course to evaluate for response to treatment, need for field/volume reduction and to assess for tolerability and toxicity. Attempts were made to reduce the field size if significant tumor regression was noted.

Chemotherapy. Systemic therapy was delivered intravenously and the agent selected was at the discretion of the treating medical oncologist. Most patients $(n=18)$ received Carboplatin (AUC2). This was administered IV and delivered 1 hour before the first fraction of each radiation cycle. The minority of patients $(n=3)$ received Cetuximab, with $250-\mathrm{mg} / \mathrm{m}^{2}$ infusion given before the first fraction of each radiation cycle.

Evaluation of palliation. Treatment-related toxicity, performance status and tumor responses were assessed every three weeks while on study and during each follow-up visit until patients died or were no longer able to comply. Tumor response was graded by the WHO criteria (23). It was assessed every three weeks by physical exam, including flexible nasopharygolaryngoscopy as indicated, and cone 
Table II. Individual patient characteristics.

\begin{tabular}{lllccc}
\hline Patient & Age & Race & Primary site & Stage & Initial presentation \\
\hline 1 & 89 & Latino & Oral cavity & III & Pain, dysphagia \\
2 & 62 & Latino & Oral cavity & IVA & Dysphagia \\
3 & 90 & Latino & Hypopharynx & IVA \\
4 & 89 & Asian & Oral cavity & IVA \\
5 & 72 & Black & Hypopharynx & III & Pain, bleeding mass \\
6 & 61 & Asian & Nasopharynx & IVB & Bleeding mass \\
7 & 59 & White & Hypopharynx & IVB & Unresectable, encasing carotids \\
8 & 50 & White & Oropharynx & Dysphagia \\
9 & 54 & Latino & Hypopharynx & IVA & Respiratory distress, emergency trach \\
10 & 61 & White & Unknown primary & IVC & Pain \\
11 & 62 & Latino & Oral cavity & Recurrent & Pain \\
12 & 62 & Latino & Larynx & IVA & Hoarseness, dysphagia \\
13 & 61 & White & Oropharynx & IVC & Bysphagia \\
14 & 90 & White & Skin H\&N & Recurrent & Neck mass \\
15 & 59 & White & Oropharynx & IVC & Pain \\
16 & 62 & Black & Oropharynx & IVC & Dysphagia \\
17 & 88 & Latino & Hypopharynx & IVC & Dysphagia \\
18 & 86 & Black & Oropharynx & IVA & Pain \\
19 & 63 & Black & Oropharynx & Recurrent & Pain, hoarseness \\
20 & 63 & Black & Oropharynx & Recurrent & Pain \\
21 & 82 & Asian & Oropharynx & IVC &
\end{tabular}

beam CT. Appropriate radiographic studies were performed 6-8 weeks after treatment completion and then later at the discretion of the treating physician. A complete response (CR) was defined as complete resolution of disease within the treatment volumes demonstrated on physical exam and radiographic imaging. A partial response (PR) was defined as $\geq 50 \%$ decrease in the volume of all gross disease within the treatment volume. No response (NR) was defined as decrease in tumor volume by $<25 \%$ or increased by $<25 \%$. Progressive disease was defined as $\geq 25 \%$ increase in tumor volume within the treatment volume.

Palliation of symptoms was scored by the treating physician and patient. Symptoms were considered to be completely palliated if the patient had no further complaint or did not require medications to control the symptom. A reduction, but not resolution of symptom severity or a decrease in pain medicine necessary to control the symptom was considered partial palliation.

Pre-treatment, on-treatment and post-treatment symptoms were documented using the Common Toxicity Criteria for Adverse Events (CTCAE) version 4.0. Subjective tolerance was documented via a "Distress Thermometer," which allowed patients to rate their global discomfort.

Statistical analysis. The clinical endpoints examined included symptom palliation, tumor response, disease-free survival (DFS) overall Survival (OS), patterns of failure, and treatment tolerance. Time to relapse was based at last clinical assessment. All relapses after radiation therapy were scored regardless of whether they were at the initial or in a secondary site of recurrence. Data were analyzed using SPSS statistical software (version 18.0; SPSS, Inc., Chicago, IL, USA). Survival analysis was carried out using the Kaplan-Meier method. Prognostic factors were analyzed using Cox ProportionalHazards model. The chi-square test was used to evaluate association between palliative response and number of treatment cycles. Prognostic and clinical factors evaluated included tumor primary site, stage, type of chemotherapy, number of treatment cycles, and palliative response. All statistical tests were based on a 2-sided hypothesis tests, $p$-Values $<0.05$ were considered to be significant.

\section{Results}

Twenty-one patients were enrolled on the treatment protocol, six patients had metastatic disease, five had recurrent disease and two patients received previous head and neck radiation. Patients were followed for a median time of 3.7 months (range 0.14-19.6). The median age of the patients in this study was 62 (range 50-90). Majority of patients had oropharyngeal $(n=8)$ or hypopharyngeal $(n=5)$ primaries. The predominant presenting symptoms were pain and dysphagia. Patient characteristics are detailed in Tables II and III.

Radiation therapy. All evaluated patients received at least one cycle of treatment. 21 patients started their second course of treatment, with sixteen patients completing all three cycles (Table IV). 15 patients were treated with IMRT technique and 6 patients received 3D conformal therapy.

Chemotherapy. All patients received radiosensitizing chemotherapy prior to each QUAD-Shot cycle. The majority of patients received carboplatin $(n=18)$ and the remaining three received cetuximab based on decision of the treating medical oncologist. 
Table III. Group characteristics.

\begin{tabular}{lccc}
\hline Characteristic & Category & N & $\%$ \\
\hline Age & Median & 62 & \\
Gender & Range & $50-80$ & \\
\multirow{3}{*}{ Tumor Site } & Male & 17 & 80.1 \\
& Female & 4 & 19.9 \\
& Oropharynx & 8 & 38.1 \\
& Hypopharynx & 5 & 23.8 \\
& Oral Cavity & 4 & 19.0 \\
& Nasopharynx & 2 & 9.5 \\
Tumor Stage & Unknown Primary & 1 & 4.8 \\
& Cutaneous & 1 & 4.8 \\
& III & 2 & 9.5 \\
& IVA & 4 & 19.9 \\
& IVB & 5 & 23.8 \\
& IVC (M1) & 6 & 28.6 \\
& Recurrent & 4 & 19.9 \\
\hline
\end{tabular}

Response to treatment. In patients $85.7 \% \quad(\mathrm{n}=18)$ had objective response to therapy with five patients $(23.8 \%)$ demonstrating clinical complete response (CR) and thirteen patients $(61.9 \%)$ demonstrating partial response (PR). Four of the five complete responders received all three courses and one patient received two courses. Ten of the partial responders received the full course of treatment. Two patients had less than a PR. Both received only 2 cycles due to refusal to continue treatment. By the chi-square test, achieving a palliative response was significantly associated with the number of applied QUAD-Shot cycles $(p=0.049)$. There was no association of response to treatment with respect to tumor primary site, stage or type of chemotherapy.

Survival. Median overall survival was seven months (range 3.710.4 months) and median progression-free survival was four months (range 1.0-8.4 months) Figures 1 and 2. Thus patients had their disease controlled for $57.1 \%$ of their remaining lifetime. In the Cox Proportional Hazards model, palliative response $(p=0.049)$ and number of QUAD-Shot cycles $(p=0.03)$ were significantly associated with improved OS.

Toxicity. The treatment was tolerated well without any grade 3 acute toxicity. Seven patients $(34.8 \%)$ had grade 2 acute toxicity, which was evenly split between grade 2 mucositis and grade 2 xerostomia. All patients had at least one grade 1 toxicity that was limited to dermatitis, mucositis or xerostomia. Two patients reported late grade 2 xerostomia and dysgeusia.

Palliation. Most patients achieved palliation of their presenting complaint, and reported subjective improvement in their quality of life. Palliation of the presenting symptom was achieved in all (100\%) of the sixteen patients that completed all three cycles. Pain severity was improved in all
Table IV. Radiation dose received.

\begin{tabular}{lccc}
\hline Dose $(\mathrm{Gy})$ & \# cycles & $\mathrm{N}$ & $\%$ \\
\hline 44.4 & 3 & 16 & 76.2 \\
29.6 & 2 & 5 & 23.8 \\
\hline
\end{tabular}

those who presented with a complaint of pain, resulting in an objective decrease in the use of opiate analgesia.

\section{Discussion}

Optimal therapy for patients with head and neck cancer not amenable to curative intent radical therapy is not defined, and treatment recommendations must account for multiple factors, including age, performance status, current and anticipated symptom burden, and individual patient goals. These patients often present with substantial tumor burden resulting in significant symptoms that negatively impact their quality of life during their limited longevity. In this series we report good response rates and symptom palliation with hypofractionated palliative local radiotherapy utilizing the QUAD-Shot regimen with concurrent radiosensitizing chemotherapy. This approach was well-tolerated with a high degree of objective tumor response $(85.7 \%)$, palliation of presenting symptoms (100\%) and minimal treatment-related morbidity. Our data is favorable compared to that reported in the literature.

Paris et al. of the University of Louisville first reported prospective data utilizing QUAD-Shot fractionation with no chemotherapy in patients with head and neck malignancies in 1992 (24). 39 lesions in 37 radiation-naïve patients ineligible for curative treatment were treated using Co-60 or $6 \mathrm{MV}$ photons using parallel opposed fields to tumor volume(s) with $1-2 \mathrm{~cm}$ margin. Field reductions were performed in the context of tumor regression and limiting dose to the spinal cord. $56.7 \%$ (21/37) of patients completed all three courses. Median OS was 4.5 months with a subjective improvement of symptoms in $85 \%$, and objective response rate of $77 \%(28 \% \mathrm{CR}, 51 \% \mathrm{PR})$ corresponding to a local control rate of $81.8 \%$ in complete responders $(9 / 11$ patients). Toxicity data was not quantified.

A decade later Peter MacCallum Cancer Centre published their prospective phase II trial using the same fractionation (4). 30 patients with head and neck primary malignancies received radiotherapy to symptomatic and large volume disease with $2 \mathrm{~cm}$ margin utilizing parallel opposed technique, with dose prescribed to the midplane. The majority of patients (29) had Stage IV disease; of those 20 were stage IVA, four stage IVB and five stage IVC with a small volume of metastatic disease. $53 \%$ of patients completed 3 courses, with an even breakdown of the remaining patients completing two or one course, respectively. Median OS was 5.7 months 


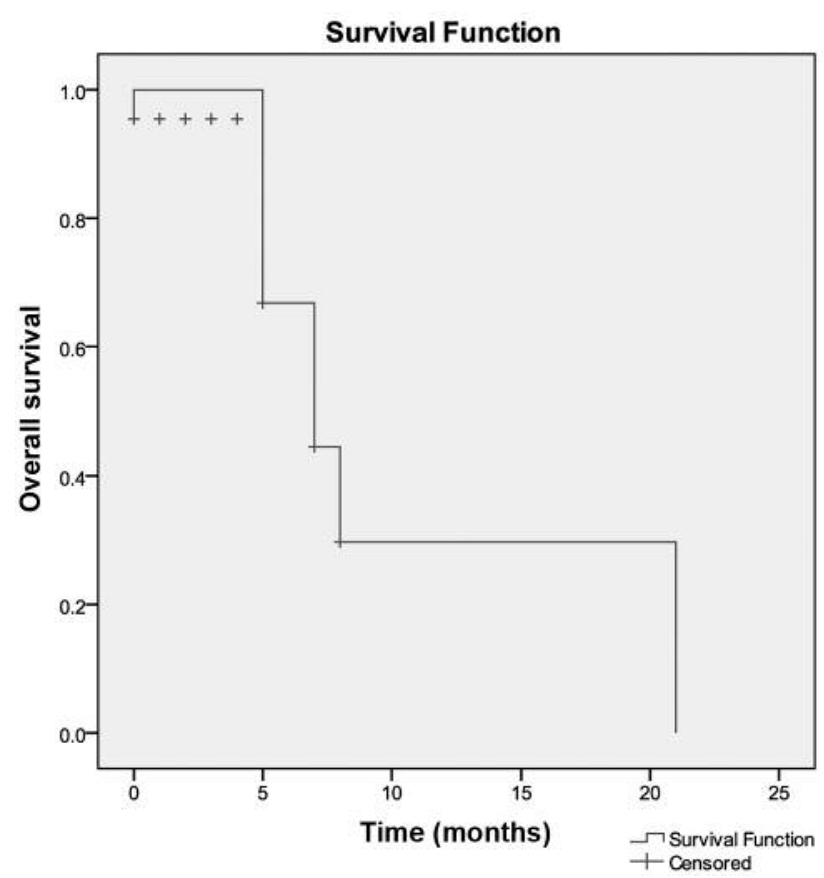

Figure 1. Overall survival.

with PFS of 3.1 months in the entire cohort, with $85 \%$ subjective symptom improvement and objective response in $53 \%$ (6.7\% CR, 46.7\% PR). Treatment was well-tolerated without reported grade 3 toxicity.

A review from UC Davis comparing various palliative radiation fractionation schemes was published shortly thereafter (1). Chen et al. performed a retrospective analysis of 60 patients treated at their institution with primary head and neck malignancies that were treated with various regimens: 44.4/12 (Quad-Shot), 70/35, 30/10, 37.5/15 and $20 / 5$. While data were not powered to detect differences in palliative response (83\% for Quad-shot vs. 77\%, 67\%, 86\% and $60 \%$ respectively), toxicity outcomes were superior for the Quad-Shot regimen with $9 \%$ grade 3 toxicity compared with $37 \%$ with the other schedules.

Given the excellent response to QUAD-Shot regimen, Carrascosa et al. pursued a prospective phase II trial investigating the addition of the radiosensitizer paclitaxel in an attempt to improve response rates and palliation of symptoms (9). Thirteen patients with pelvic and 7 patients with head and neck primary malignancy were treated with Quad Shot radiation with paclitaxel $\left(60 \mathrm{mg} / \mathrm{m}^{2}\right)$ delivered prior to each RT cycle. Radiation was delivered to symptomatic primary disease and wired palpable nodes using parallel opposed fields with $2 \mathrm{~cm}$ margins and similar field reductions. The entire cohort had a response rate of $94.7 \%$ (26.3\% CR, 68.4\% PR) with $89.5 \%$ experiencing symptom

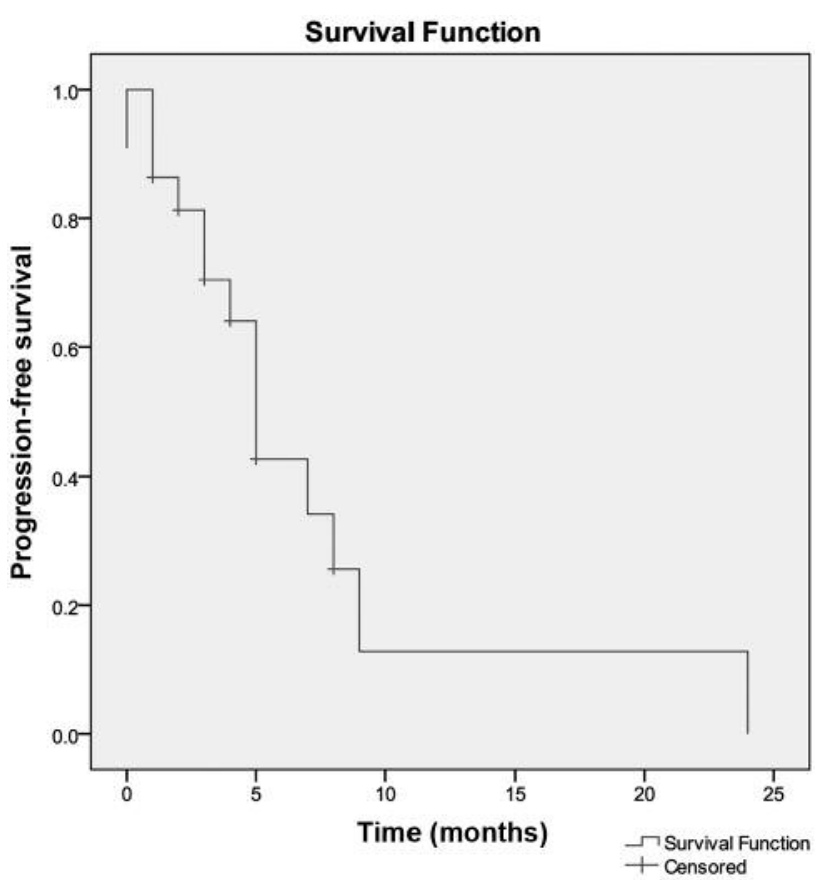

Figure 2. Progression-free survival.

palliation (57.9\% complete, $31.9 \%$ partial) for a median duration of 36 weeks. Treatment was well-tolerated with one patient with grade 3 anemia, another with grade 3 mucositis and two with grade 2 allergic reactions to paclitaxel. Restricting analysis for $6 / 7$ evaluable head and neck patients, $1 / 6$ had complete tumor response and with 3/6 each having complete or partial symptom relief. Median survival was 33 weeks for the entire cohort and 28 weeks for the head and neck patients.

Memorial Sloan Kettering Cancer Center reported a series of seventy-five patients with head and neck incurable primaries or metastatic cancers treated with the RTOG 8502 regimen. Twenty-eight (37\%) patients completed at least 3 cycles of the regimen. At the discretion of the treating medical oncologist, some patients received concurrent chemotherapy, defined as administered within 7 days of the radiation. Sixty-five percent had a palliative response with a median overall survival of 5.67 months (25). Our series included a larger proportion of patients with metastatic or recurrent disease, as well as two with prior head and neck radiation and stricter criteria for the administration of radiosensitizing chemotherapy.

Recently, Seiwert et al. reported the results of their phase $1 \mathrm{~b}$ trial using pembrolizumab for recurrent (not amenable to locally curative treatment) or metastatic squamous cell carcinoma of the head and neck. All patients had ECOG performance status of $0-1$ and adequate organ function to 
receive the treatment. The overall response rate confirmed by imaging with the PD-1 inhibitor was $18 \%$, with $17 \%$ of the patients having grade 3-4 drug toxicity. Overall, $82 \%$ of the patients discontinued treatment because of progressive disease and/or adverse events. The median progression free survival in their study was 2 months (26).

Despite including patients with advanced disease burden, limited longevity and at higher risk for complications, we report prolonged median and progression free survival of seven and four months, respectively. In patients $57 \%$ had their disease effectively-controlled during their remaining lifetime. Every patient received palliation of their symptoms without a single grade 3 toxicity. All patients were treated by physicians and staff specifically trained in the management of head and neck cancer. Unlike previous reports, we utilized modern radiotherapy techniques with the majority of patients treated with intensity modulated and image guided radiation therapy (IMRT and IGRT). This may have allowed for superior tumor coverage with decreased PTV expansions, hence limiting irradiation of normal tissue and toxicity. With required routine reductions of the spinal cord in the cited literature using $2 \mathrm{D}$ or $3 \mathrm{D}$ technique, coverage of GTV may have been compromised. Significant responses were even seen in patients with recurrent disease who may have inherently more resistant tumors, as well as patients with metastatic disease. Safety of QUAD-Shot chemoradiation was also demonstrated in a pair of patients that received prior radiation to overlapping treatment volumes. $76.2 \%(16 / 21)$ of patients were able to complete all three courses, indicating the tolerability and feasibility of the treatment, and related to a greater palliative treatment response and better outcomes.

The present study has the inherent limitations of a retrospective analysis. It also lacks an objective symptom assessment and relied on subjective patient and physician reports. Given the small proportion of patients who present with disease ineligible for radical therapy, we have a limited sample size, which is comparable to the previous literature.

\section{Conclusion}

Palliative QUAD-Shot hypofractionated radiotherapy in combination with radiosensitizing chemotherapy may yield better than anticipated outcomes compared to historical-used regimens with higher rates of tumor response and effective symptom relief, with acceptable toxicity. A future study in a prospective manner with incorporation of biomarkers such as HPV status is warranted.

\section{References}

1 Chen AM, Vaughan A, Narayan S and Vijayakumar S: Palliative radiation therapy for head and neck cancer: toward an optimal fractionation scheme. Head Neck 30: 1586-1591, 2008.
2 Al-Mamgani A, Tans L, Van rooij PH, Noever I, Baatenburg de jong RJ and Levendag PC: Hypofractionated radiotherapy denoted as the "Christie scheme": an effective means of palliating patients with head and neck cancers not suitable for curative treatment. Acta Oncol 48: 562-570, 2009.

3 Agarwal JP, Nemade B, Murthy V, Ghosh-Laskar S, Budrukkar A, Gupta T, D'Cruz A, Pai P, Chaturvedi P and Dinshaw K: Hypofractionated, palliative radiotherapy for advanced head and neck cancer. Radiother Oncol 89: 51-56, 2008.

4 Corry J, Peters LJ, Costa ID, Milner AD, Fawns H, Rischin D and Porceddu S: The 'QUAD SHOT' - a phase II study of palliative radiotherapy for incurable head and neck cancer. Radiother Oncol 77: 137-142, 2005.

5 Porceddu SV, Rosser B, Burmeister BH, Jones M, Hickey B, Baumann K, Gogna K, Pullar A, Poulsen M and Holt T: Hypofractionated radiotherapy for the palliation of advanced head and neck cancer in patients unsuitable for curative treatment - "Hypo Trial". Radiother Oncol 85: 456-462, 2007.

6 Stevens CM, Huang SH, Fung S, Bayley AJ, Cho JB, Cummings BJ, Dawson LA, Hope AJ, Kim JJ, O'Sullivan B, Waldron JN and Ringash J: Retrospective study of palliative radiotherapy in newly diagnosed head and neck carcinoma. Int J Radiat Oncol Biol Phys 81: 958-963, 2011.

7 Kancherla KN, Oksuz DC, Prestwich RJ, Fosker C, Dyker KE, Coyle CC and Sen M: The role of split-course hypofractionated palliative radiotherapy in head and neck cancer. Clin Oncol ( $R$ Coll Radiol) 23: 141-148, 2011.

8 Spanos W Jr, Guse C, Perez C, Grigsby P, Doggett RL and Poulter C: Phase II study of multiple daily fractionations in the palliation of advanced pelvic malignancies: preliminary report of RTOG 8502. Int J Radiat Oncol Biol Phys 17: 659-661, 1989.

9 Carrascosa LA, Yashar CM, Paris KJ, Larocca RV, Faught SR and Spanos WJ: Palliation of pelvic and head and neck cancer with paclitaxel and a novel radiotherapy regimen. J Palliat Med 10: 877-881, 2007.

10 Vermorken JB, Trigo J, Hitt R, Koralewski P, Diaz-Rubio E, Rolland F, Knecht R, Amellal N, Schueler A and Baselga J: Open-label, uncontrolled, multicenter phase II study to evaluate the efficacy and toxicity of cetuximab as a single agent in patients with recurrent and/or metastatic squamous cell carcinoma of the head and neck who failed to respond to platinum-based therapy. J Clin Oncol 25: 2171-2177, 2007.

11 Forastiere AA, Shank D, Neuberg D, Taylor SG 4th, DeConti RC and Adams G: Final report of a phase II evaluation of paclitaxel in patients with advanced squamous cell carcinoma of the head and neck: an Eastern Cooperative Oncology Group trial (PA 390). Cancer 82: 2270-2274, 1998.

12 Samlowski WE, Moon J, Kuebler JP, Nichols CR, Gandara DR, Ozer H, Williamson SK, Atkins JN, Schuller DE and Ensley JF: Evaluation of the combination of docetaxel/carboplatin in patients with metastatic or recurrent squamous cell carcinoma of the head and neck (SCCHN): a Southwest Oncology Group Phase II study. Cancer Invest 25: 182-188, 2007.

13 Forastiere AA, Metch B, Schuller DE, Ensley JF, Hutchins LF, Triozzi P, Kish JA, McClure S, VonFeldt E, Williamson SK and Von Hoff DD: Randomized comparison of cisplatin plus fluorouracil and carboplatin plus fluorouracil versus methotrexate in advanced squamous-cell carcinoma of the head and neck: a Southwest Oncology Group study. J Clin Oncol 10: 1245-1251, 1992. 
14 Vermorken JB, Mesia R, Rivera F, Remenar E, Kawecki A, Rottey S, Erfan J, Zabolotnyy D, Kienzer HR, Cupissol D, Peyrade F, Benasso M, Vynnychenko I, De Raucourt D, Bokemeyer C, Schueler A, Amellal N and Hitt R: Platinumbased chemotherapy plus cetuximab in head and neck cancer. $\mathrm{N}$ Engl J Med 359: 1116-1127, 2008.

15 Monnier L, Touboul E, Durdux C, Lang P, St Guily JL and Huguet F: Hypofractionated palliative radiotherapy for advanced head and neck cancer: the IHF2SQ regimen. Head Neck 35: 1683-1688, 2013.

16 Coughlin CT and Richmond RC: Biologic and clinical developments of cisplatin combined with radiation: concepts, utility, projections for new trials, and the emergence of carboplatin. Semin Oncol 16(4 Suppl 6): 31-43, 1989.

17 Elomaa L, Joensuu H, Kulmala J, Klemi P and Grenman R: Squamous cell carcinoma is highly sensitive to taxol, a possible new radiation sensitizer. Acta Otolaryngol 115: 340-344, 1995.

18 Preisler VK, Stopper H, Schindler D, Friedl R, Pfreundner L, Hoppe $\mathrm{F}$ and Hagen R: Cytotoxic and genotoxic effects of paclitaxel (Taxol) and radiation in a squamous cell carcinoma cell line of the larynx. Acta Otolaryngol 118: 600-605, 1998.

19 Douple EB, Richmond RC, O'Hara JA and Coughlin CT: Carboplatin as a potentiator of radiation therapy. Cancer Treat Rev 12(Suppl A): 111-124, 1985.

20 Yang LX, Douple EB, O'Hara JA and Wang HJ: Production of DNA double-strand breaks by interactions between carboplatin and radiation: a potential mechanism for radiopotentiation. Radiat Res 143: 309-315, 1995.

21 Muggia FM: Overview of carboplatin: replacing, complementing, and extending the therapeutic horizons of cisplatin. Semin Oncol 16(2 Suppl 5): 7-13, 1989.
22 Volling P, Staar S, Achterrath W and Muller RP: Carboplatin plus radiation therapy in head and neck cancer. Semin Oncol 18(1 Suppl 2): 17-22, 1991.

23 Therasse P, Arbuck SG, Eisenhauer EA, Wanders J, Kaplan RS, Rubinstein L, Verweij J, Van Glabbeke M, Van Oosterom AT, Christian MC and Gwyther SG: New guidelines to evaluate the response to treatment in solid tumors. J Natl Cancer Inst 92: 205-216, 2000.

24 Paris KJ, Spanos WJ Jr., Lindberg RD, Jose B and Albrink F: Phase I-II study of multiple daily fractions for palliation of advanced head and neck malignancies. Int J Radiat Oncol Biol Phys 25: 657-660, 1993.

25 Lok BH, Jiang G, Gutiontov S, Lanning RM, Sridhara S, Sherman EJ, Tsai CJ, McBride SM, Riaz N and Lee NY: Palliative head and neck radiotherapy with the RTOG 8502 regimen for incurable primary or metastatic cancers. Oral Oncol 51: 957-962, 2015.

26 Seiwert TY, Burtness B, Mehra R, Weiss J, Berger R, Eder JP, Heath K, McClanahan T, Lunceford J, Gause C, Cheng JD and Chow LQ: Safety and clinical activity of pembrolizumab for treatment of recurrent or metastatic squamous cell carcinoma of the head and neck (KEYNOTE-012): an open-label, multicentre, phase 1b trial. Lancet Oncol 17: 956-965, 2016.

Received January 5, 2017

Revised January 27, 2017

Accepted January 31, 2017 